\title{
Numerical simulations of blood cell flow in non-Newtonian fluid
}

\author{
Kazuhiro Shitara ${ }^{1}$, and Toru Hyakutake, \\ ${ }^{1}$ Graduate School of Engineering, Yokohama National University, 240-8501 Yokohama, Japan \\ ${ }^{2}$ Faculty of Engineering, Yokohama National University, 240-8501 Yokohama, Japan
}

\begin{abstract}
We investigated how non-Newtonian viscosity behavior affects the flow characteristics of blood cells. Our findings offer insight about how shear thinning affects the dispersion of liposome-encapsulated hemoglobin and red blood cells in blood. The lattice Boltzmann method was used for fluid calculations, and the rheological properties of the non-Newtonian fluid were modeled with power-law relationships. The deformable three-dimensional red blood cell model was applied. First, we investigated the effects of shear thinning on the flow behavior of single blood cell. Simulation results indicate that shear thinning promotes the axial concentration of red blood cells. Next, varied the hematocrit to see how mutual interference between blood cells affects flow. At low hematocrit, shear thinning clearly promotes the axial concentration of red blood cells. As the hematocrit increases, in contrast, mutual interference has a greater effect, which counteracts shear thinning so the red blood cell distribution resembles the distribution within a Newtonian fluid.
\end{abstract}

\section{Introduction}

Red blood cells (RBCs) play an essential role in supplying oxygen to all tissues in the body. When the diameters of RBCs and vessels are on the same order of magnitude, the rheological properties of RBCs greatly influence blood flow. RBCs are biconcave and diskshaped, and they deform passively under external force. This deformability has some effect on RBCs' tendency to concentrate along the axis of flow paths, which helps them to flow smoothly through narrow microvessels.

Several recent numerical studies have focused on the behavior of deformable RBCs in microvascular flows [19]. The motion of RBCs is described well with existing numerical techniques. However, most studies have focused on the flow of RBCs in the Newtonian fluid of blood plasma. Currently, liposome-encapsulated hemoglobin (LEH) is being promoted vigorously in Japan as synthetic alternative to RBCs [10]. Typical LEH particles are $200-250 \mathrm{~nm}$ in diameter, which is $1 / 30$ smaller than a human RBC. A solution of plasma and LEH shows non-Newtonian behavior in some conditions [11]. To better understand this behavior, we have simulated the flow of surrounding plasma-LEH solution on the flow of erythrocytes in microvessels. The simulation results offer valuable insights into the mechanisms by which the flow of RBCs delivers oxygen through microvascular networks.

\section{Numerical method}

We constructed a three-dimensional microvascular model and investigated the flow behavior of RBCs in the
Newtonian and non-Newtonian fluid. Figure 1 shows schematics of the microvascular model we employed, in which the vessel diameter $(D)$ is $20 \mu \mathrm{m}$ and the vessel length $\left(L_{\mathrm{x}}\right)$ is $40 \mu \mathrm{m}$. The mean velocity in the vessel $U_{\mathrm{m}}$ was set to $1.5 \times 10^{-3} \mathrm{~m} / \mathrm{s}$. Three hematocrit levels were simulated, at $3.0 \%, 6.0 \%$ and $15 \%$. Blood flow was simulated using the lattice Boltzmann method (LBM) [12] in conjunction with the immersed boundary method (IBM) [13] to account for fluid-membrane interactions between the flow field and deformable RBCs. Guo's method was adopted to account for spatiotemporal changes in forces on the flow and to model unsteady flows accurately [14]. At the upstream and downstream boundaries, periodic boundary conditions with a pressure difference were imposed. The no-slip boundary condition was applied to the boundary nodes to represent that the flow velocity at the wall matches the velocity of the wall.

To model RBCs, the unstressed structure was given the biconcave shape proposed by Evans and Fung [15]. Each RBC membrane was modeled with 1,272 triangular elements. Membrane forces due to strain, bending,
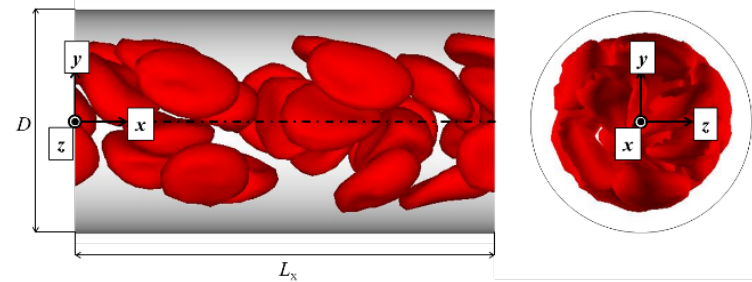

Fig. 1. Numerical model

\footnotetext{
Corresponding author: hyaku@ynu.ac.jp
} 
surface, and volume constraints were computed from the corresponding energy terms using the principle of virtual work [16]. Reference values were used to determine the parameters of the RBC membrane [17]. Viscosity inside the RBCs was assumed to be five times greater than the viscosity of plasma without LEH.

The surrounding fluid was modeled as both plasma without LEH (Newtonian) and plasma with LEH (nonNewtonian). For non-Newtonian fluid, we applied the following power-law model to describe the fluid viscosity,

$$
\mu=\mu_{1} \gamma^{n-1}
$$

where $\mu$ is the apparent viscous coefficient, $\gamma$ is the shear rate, and $n$ is an index that distinguishes non-

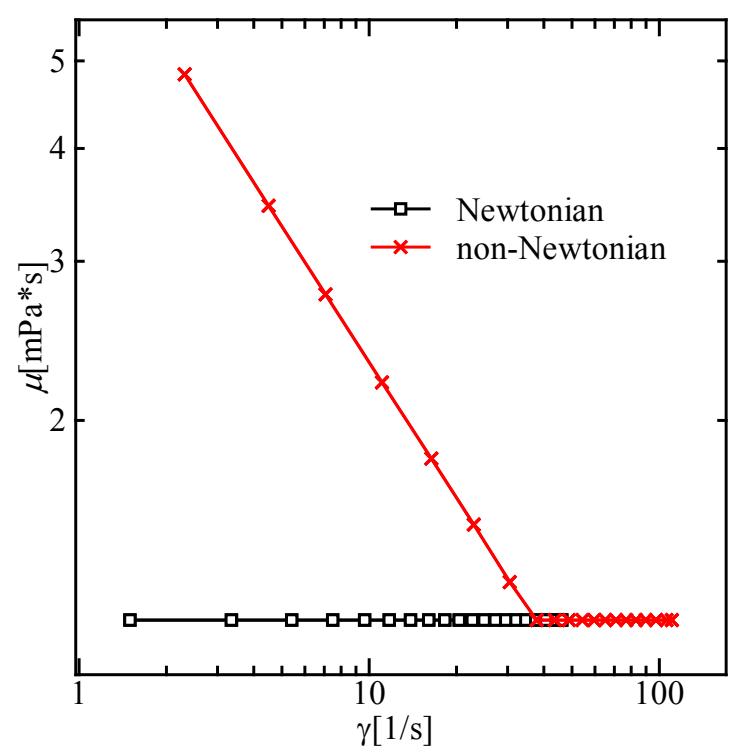

Fig. 2. Relationship between shear rate and viscosity

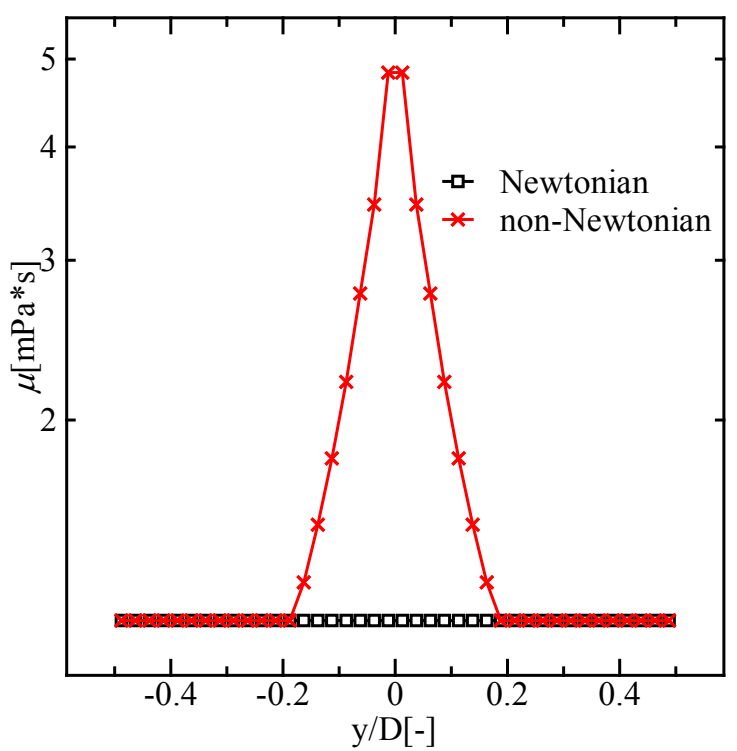

Fig. 3. Viscous distribution in the microvessel
Newtonian and Newtonian fluids. $\mu_{1}$ means the viscous coefficient at $n=1.0$ (Newtonian fluid). Figure 2 plots the relationship between shear rate and the viscosity of the surrounding fluid that follows from Eq. 1. For the non-Newtonian fluid, we assumed $n=1$ and $\mu_{1}=1.2$ $\mathrm{mPa} \cdot \mathrm{s}$. Figure 3 shows the distribution of viscosity in the microvessel. The viscosity of the Newtonian fluid was constant in the vessel. For the non-Newtonian fluid, in contrast, the high-viscosity fluid was concentrated around the vessel axis since the flow velocity was low near the axis. This high-viscosity region was approximately five times more viscous than the fluid near the vessel walls.

\section{Results and discussion}

\subsection{Single RBC}

First, we calculated the flow of single RBC in Newtonian and non-Newtonian fluids. Figure 4 shows the trajectories of the center of gravity of the simulated RBCs. We considered two initial positions with $y_{0}$ set to 2.0 and $5.0 \mu \mathrm{m}$. The position of $y_{0}=5.0 \mu \mathrm{m}$ is the midpoint between the vessel centerline $(y=0 \mu \mathrm{m})$ and wall $(y=10 \mu \mathrm{m})$. As time passes, the $y$-coordinates of the RBCs changed. Shear thinning in the non-Newtonian fluid tended to promote the axial concentration of RBCs. In a non-Newtonian fluid, viscosity is higher at the center of the vessel. In this simulation, since the mean velocity was constant, the capillary number $(\mathrm{Ca}=$ $\left.\mu \mathrm{U}_{\mathrm{m}} / D E_{\mathrm{s}}\right)$ increases toward the vessel center, where $E_{\mathrm{s}}$ is the strain modulus of the RBC membrane. A higher capillary number causes larger deformation of the RBC. The associated variation in the viscosity in the vessel encourages the RBCs to cluster around the vessel center.

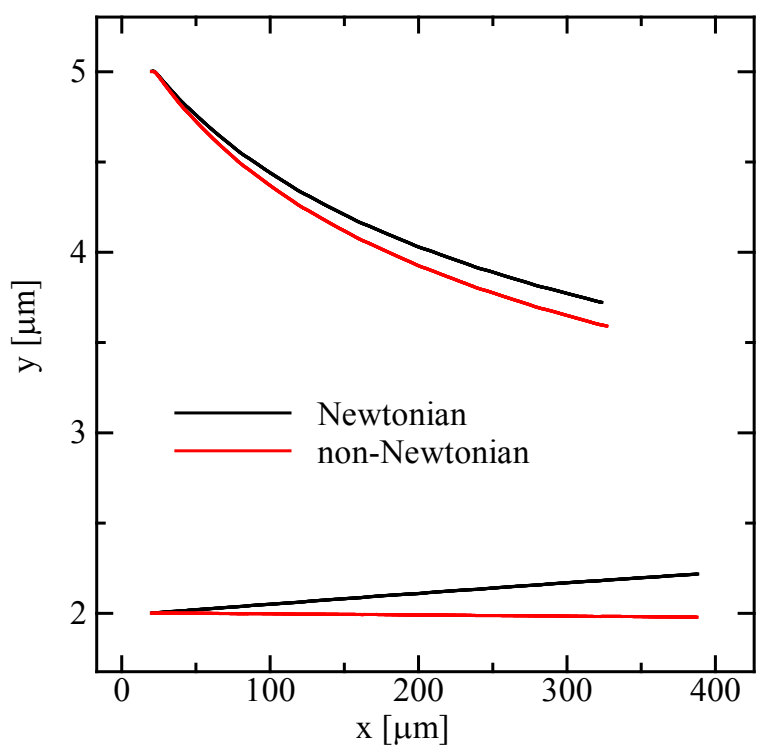

Fig. 4. Trajectories of single RBC 


\subsection{Multiple RBCs}

Next, we calculated the flow of multiple RBCs. Figure 5 plots the RBC flow for the three hematocrit values we simulated. Figure 6 shows the time average of the RBC distribution in the vessel. The horizontal axis gives the distance from the vessel centerline, and the vertical axis indicates the area ratio of $\mathrm{RBC}$ passing through the downstream cross-section. These results show that a cellfree layer formed due to the axial concentration of RBCs. This cell-free layer became thicker as the hematocrit decreased. In the case of $\mathrm{Hct}=3.0 \%$, the $\mathrm{RBC}$ distributions in Newtonian and non-Newtonian fluids are very different. With small hematocrit values, shear thinning dominates the axial concentration of RBCs. As the hematocrit increases, mutual interference between RBCs has more of an effect, so the distribution of RBCs is less different between Newtonian and non-Newtonian fluids. When Hct $=6.1 \%$, the RBCs were more clustered near $r=4 \mu \mathrm{m}$ in non-Newtonian fluid than in Newtonian fluid. To investigate this phenomenon in more detail, we defined the orientation angle, which is the angle between $x$-direction and the longitudinal direction of the RBC. We recorded the orientation angles

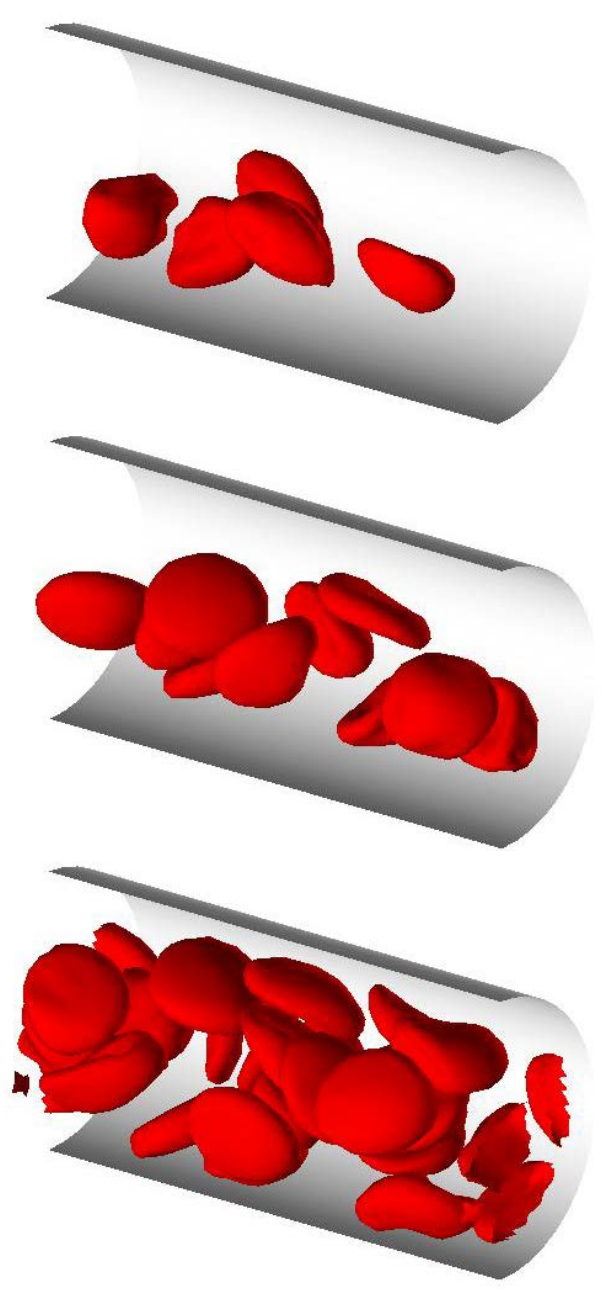

Fig. 5. Image of RBC flow for various hematocrit (upper : Hct $=3.0 \%$, middle $:$ Hct $=6.1 \%$, lower $:$ Hct $=15 \%$ )
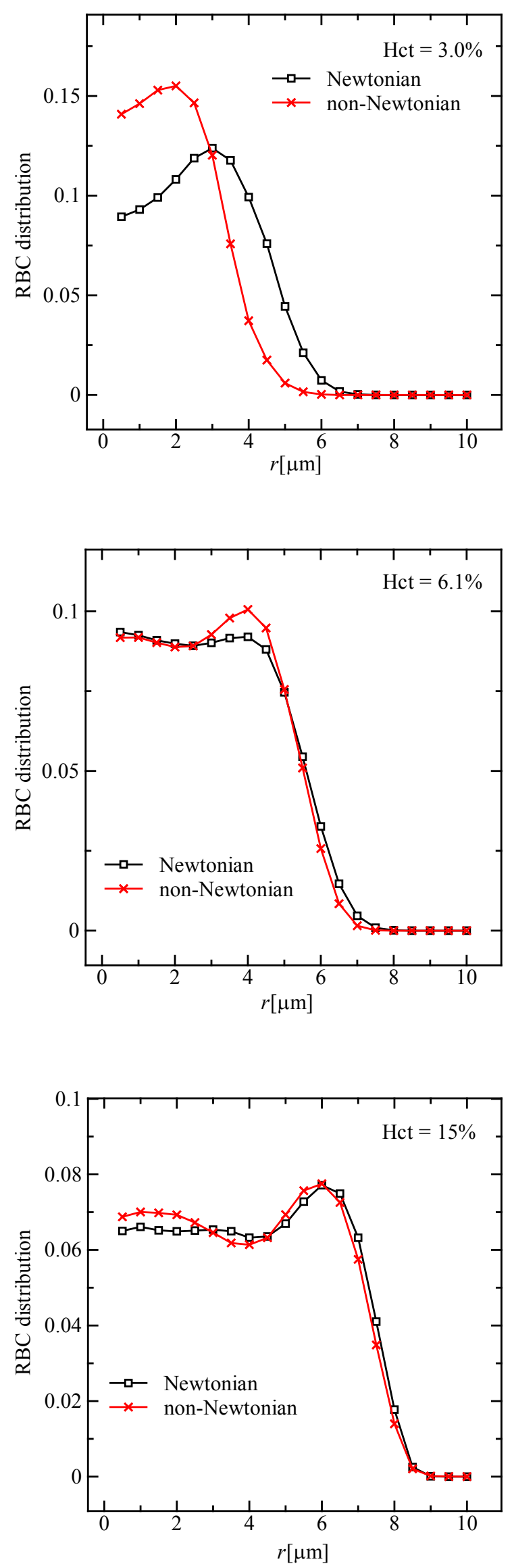

Fig. 6. RBC distributions for various hematocrit (upper : Hct $=3.0 \%$, middle $:$ Hct $=6.1 \%$, lower $:$ Hct $=15 \%$ ) 


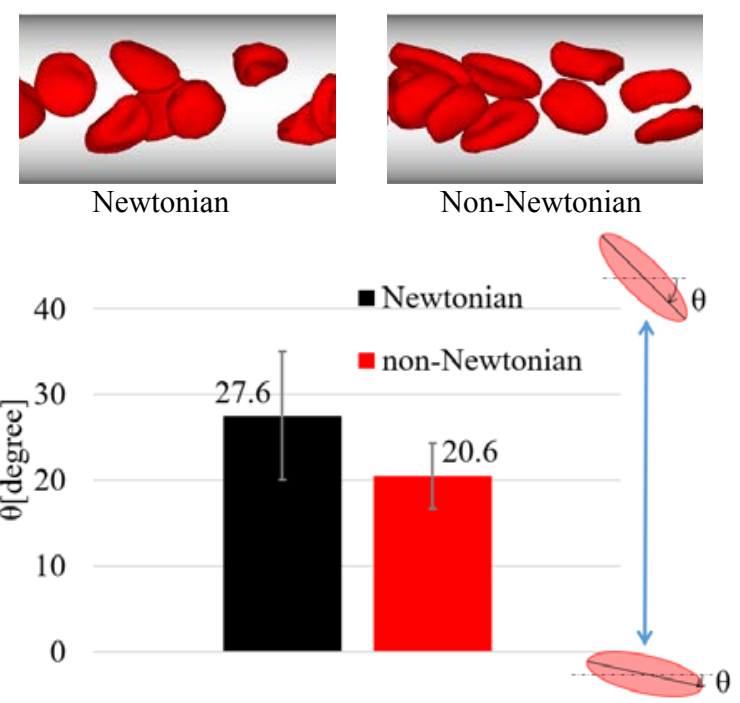

Fig. 7. Comparison of the orientation angle between Newtonian and non-Newtonian fluids

of the RBCs using image analysis. In Fig. 7, the RBC flow images and the orientation angles are compared between Newtonian and non-Newtonian fluids at Hct $=$ $6.1 \%$. The angles for non-Newtonian fluid tended to be larger than those in Newtonian fluids. This explains the concentration of RBCs around $r=4 \mu \mathrm{m}$. On the other hand, with Hct $=15 \%$, the difference in the orientation angles almost disappeared.

\section{Conclusion}

In this study, to clarify the flow behavior of RBCs in blood with added LEH, the effects of shear thinning on the flow in blood vessels were investigated using threedimensional microvasucular models. First, we investigated the flow behavior of single blood cell in Newtonian and non-Newtonian fluids. The simulation results indicate that shear thinning encourages RBCs to concentrate around the vessel axis. Next, we investigated the flow behavior of multiple blood cells by varying the hematocrit. These simulation results indicate that shear thinning has more of an effect at low hematocrit levels. The orientation angle of the RBCs was finally investigated. The difference in the orientation angles in Newtonian and non-Newtonian fluids explains the variation in RBC distribution within microvessels. As the hematocrit increases further, the interactions between the RBCs have more of an effect, so the discrepancy in the distribution RBCs in Newtonian and non-Newtonian fluids nearly disappeared.

\section{References}

1. Boryczko, K., Dzwinel, W., Yuen, D.A., Dynamical clustering of red blood cells in capillary vessels, J. Mol. Model., 9 (2003), 16-33.

2. Dupin, M.M., Halliday, I., Care, C.M., Alboul, L., Munn, L.L., Modeling the flow of dense suspensions of deformable particles in three dimensions, Phys. Rev. E, Stat. Nonlin. Soft Matter Phys., 75 (2007), 066707.
3. Zhang, J., Johnson, P.C., Popel, A.S., Effects of erythrocyte deformability and aggregation on the cell free layer and apparent viscosity of microscopic blood flows, Microvasc. Res., 77 (2009), 265-272.

4. Sugiyama, K., Ii, S., Takeuchi, S., Takagi, S., Matsumoto, Y., Full Eulerian simulations of biconcave neo-Hookean particles in a Poiseuille flow, Comput. Mech., 46 (2010), 147-157.

5. Zhao, H., Isfahani, A.H.G., Olson, L.N., Freund, J.B., A spectral boundary integral method for flowing blood cells, J. Comput. Phys., 229 (2010), 3726-3744.

6. Tsubota, K., Wada, S., Effect of the natural state of an elastic cellular membrane on tank-treading and tumbling motions of a single red blood cell, Phys. Rev. E, Stat. Nonlin. Soft Matter Phys., 81 (2010), 011910.

7. Imai, Y., Kondo, H., Ishikawa, T., Teck Lim, C., Yamaguchi, T., Modeling of hemodynamics arising from malaria infection, J. Biomech., 43 (2010), 1386-1393.

8. Secomb, T.W., Mechanics and computational simulation of blood flow in microvessels, Med. Eng. Phys., 33 (2011), 800-804.

9. Hyakutake, T., Nagai, S., "Numerical simulation of red blood cell distributions in three-dimensional microvascular bifurcations", Microvasc. Res., 97 (2015), 115-123.

10. Sakai, H., Sou, K., Horinouchi, H., Kobayashi, K., Tsuchida, E., Review of hemoglobin-vesicles as artificial oxygen carriers, Artif. Organs, 33 (2009), $139-145$.

11. Sakai, H., Sato, A., Okuda, N., Takeoka, S., Maeda, N., Tsuchida, E., "Peculiar flow patterns of RBCs suspended in viscous fluids and perfused through a narrow tube $(25 \mu \mathrm{m})$ ", Am. J. Physiol.-Heart Circul. Physiol., 297 (2009), 583-589.

12. Succi, S., The lattice Boltzmann equation: for fluid dynamics and beyond, Oxford: Oxford University Press (2001).

13. Peskin, C.S., Numerical analysis of blood flow in the heart, J. Comput. Phys., 25 (1977), 220-252.

14. Guo, Z., Zheng, C., Shi, B., Discrete lattice effects on the forcing term in the lattice Boltzmann method, Phys. Rev. E, Stat. Nonlin. Soft Matter Phys., 65 (2002), 046308.

15. Evans, E.A., Fung, Y.C., Improved measurements of the erythrocyte geometry, Microvasc. Res., 4 (1972), 335-347.

16. Krüger, T., Computer simulation study of collective phenomena in dense suspensions of red blood cells under shear, Berlin: Springer (2012).

17. Oulaid, O., Saad, A.K.W., Aires, P.S., Zhang, J., Effects of shear rate and suspending viscosity on deformation and frequency of red blood cells tanktreading in shear flows, Comp. Methods Biomech. Biomed. Engin., 19 (2016), 648-662. 